\title{
Development, Validation and Application of Tandem Mass Spectrometry for Screening of Inborn Metabolic Disorders in Kuwaiti Infants
}

\author{
Mohammed Abdel-Hamid ${ }^{a}$ Klara Tisocki ${ }^{b}$ Laila Sharaf $^{a}$ Dina Ramadan ${ }^{c}$ \\ Departments of a Pharmaceutical Chemistry and ${ }^{b}$ Pharmacy Practice, Faculty of Pharmacy, Kuwait University, and \\ 'Department of Pediatrics, Sabah Hospital, Kuwait
}

\section{Key Words}

Inborn metabolic disorders · Tandem mass spectrometry • Validation study

\begin{abstract}
Objective: The aim of this work was to develop a specific and validated tandem mass spectrometric (MS/MS) method for screening of amino acidopathies, organic acidurias, urea cycle disorders and fatty acid oxidation defects in Kuwaiti newborns and sick infants. Materials and Methods: A total of 1,520 blood samples were tested for inborn metabolic disorders in Kuwaiti newborns and sick infants. Positive electrospray MS/MS was used to measure diagnostic acylcarnitines and amino acids in blood spots after simple extraction and derivatization procedures. Validation and stability studies were conducted using control blood samples supplemented with known concentrations of the diagnostic amino acids or acylcarnitines. Reference and cutoff levels of the diagnostic metabolites were determined in a group of 500 normal Kuwaiti babies for quantitative evaluation. Results: Of the 1,520 samples, 32 were positive newborn cases and 27 positive symptomatic infants. For the validation studies, the range of relative standard deviation was $2.6-14.7 \%$, whereas the range of the percent deviation from nominal concentrations was -23.0 to +25.0 of the diagnostic metabolites. Sta-
\end{abstract}

bility studies indicated appropriate stability of the diagnostic amino acids and acylcarnitines in dried blood spots stored at $22 \pm 1{ }^{\circ} \mathrm{C}$ and relative humidity of $50-60 \%$. Conclusions: Tandem mass spectrometry can significantly contribute to a newborn screening program as a fast and highly specific diagnostic technique for screening of a broad range of inborn metabolic disorders.

Copyright $\odot 2007$ S. Karger AG, Basel

\section{Introduction}

Inborn metabolic disorders (IMDs) are rare types of genetic disorders that cause irreversible mental and/or physical disability or death in affected children. Early diagnosis of IMDs can dramatically reduce the risk of death and can prevent long-term neurological complications [1, 2]. Recently, electrospray tandem mass spectrometry (MS/MS) has been described as a powerful technique for screening of IMDs. The technique permits the detection and quantification of many IMDs in a single blood spot for most fatty acid oxidation, amino acid, organic acid and urea cycle disorders. At present, MS/MS is being used as the screening technique for the diagnosis of IMDs in newborns and sick infants in many clinical biochemical laboratories in the USA, Europe, Australia and Japan [3-

\section{KARGER}

Fax +41613061234

E-Mail karger@karger.ch

www.karger.com
(C) 2007 S. Karger AG, Basel

1011-7571/07/0163-0215\$23.50/0

Accessible online at:

www.karger.com/mpp
Dr. M.E. Abdel-Hamid

Department of Pharmaceutical Chemistry, Faculty of Pharmacy, Kuwait University PO Box 24923

Safat 13110 (Kuwait)

Tel. +965968 9126, Fax +965 534 2807, E-Mail Abdel-Hamid@hsc.edu.kw 
6]. The outcomes and implications of tandem mass spectrometry in metabolic screening in comparison to other screening techniques have been reported [5]. In the Arab region, with the exception of the Kingdom of Saudi Arabia, the use of MS/MS in metabolic screening programs is at its developmental stage [7]. Presumably, this may be attributed to the high costs of equipment and consumables and lack of technical expertise. The recent development in tandem mass technology has significantly contributed to the development of automated and routinely applied methods for IMD screening [8-9]. Clinical studies have shown a high incidence rate of IMDs among the Kuwaiti population $[5,9]$. Comparable high frequencies of IMDs have been reported in the Kingdom of Saudi Arabia, Egypt and other Gulf countries [10,11]. The high prevalence of metabolic disorders is most likely due to the widespread tradition of consanguineous marriages in these regions [12].

Recently, the Kuwait Medical Genetic Center has introduced the neonatal screening service for phenylketonuria and congenital hypothyroidism in Kuwait using fluorescence immunoassay technique. This screening procedure has several limitations, e.g. a narrow range of metabolic diseases detected, non-specificity and crossreactivity of analytes, which may lead to false-positive tests.

To avoid this, only samples of severely affected infants are sent abroad for metabolic screening by MS/MS. However, this outsourcing is costly and time-consuming, particularly when the child is hospitalized in the intensive care unit. Accordingly, it was decided to use MS/MS available at the Faculty of Pharmacy, Kuwait University, for screening IMDs. Hence the objective of this study was to develop a specific and validated MS/MS method screen for IMDs in newborns and sick infants in Kuwait.

\section{Materials and Methods}

\section{Materials}

Acylcarnitines and amino acids purchased from Sigma-Aldrich (St. Louis, Mo., USA) were used as supplied. Isotopically labeled internal standards of amino acids (Set A) and acylcarnitines (Set B) were supplied by Cambridge Isotope Laboratories (Woburn, Mass., USA). Butanolic $\mathrm{HCl}$ was obtained from Regis Technologies (Morton Grove, Ill., USA). HPLC-grade methanol and acetonitrile were purchased from Fisher Scientific, Auburn, USA. Water was purified by Milli-Q device (Millipore, Bedford, Mass., USA). Guthrie filter paper cards (S\&S 903; Schleicher and Schull, Dassel, Germany) were used to collect blood samples. For validation and stability studies, certified human blood samples were donated by the Kuwait Central Blood Bank, Kuwait.
Methods

A total of 1,520 blood samples were collected between May 2004 and March 2006 from 1,158 newborns and 362 sick infants. All newborns and infants tested were cared for in maternity wards and pediatric clinics of the seven major governmental maternity and pediatrics hospitals in Kuwait. The blood samples taken by heel stick were spotted on Guthrie filter paper cards, left to dry at room temperature for at least $24 \mathrm{~h}$, placed inside plastic envelopes and then sent to the Faculty of Pharmacy, Kuwait University, for screening by MS/MS. The blood spots were analyzed for acylcarnitines and amino acids by in-house triple-quadruple tandem mass spectrometer (Quattro LC; Micromass, Manchester, UK) with a positive electrospray ionization probe. Using a BSD 400 Puncher, a single 3-mm disk ( 3 $\mu$ lblood) was punched in the center of the dried blood spot. The disk was placed in a capped glass for extraction of the blood with $200 \mu \mathrm{l}$ of methanol, containing known concentrations of a mixture of isotopically labeled internal standards of diagnostic acylcarnitines and amino acids. After $\sim 20 \mathrm{~min}$ of shaking, the solvent was gently evaporated and the residue was mixed with $\sim 80 \mu$ l of butanolic $\mathrm{HCl}$ and heated at $65^{\circ} \mathrm{C}$ for $15 \mathrm{~min}$ in a capped glass tube. The solvent was evaporated again and the residue was finally reconstituted in $80 \mu \mathrm{l}$ of the mobile phase (acetonitrile/water, $80 / 20 \mathrm{v} / \mathrm{v}$ ). A $20-\mu \mathrm{l}$ aliquot of each sample was directly injected into the mobile phase flowing to the ionization probe of the tandem mass spectrometer, at a flow rate of $0.1 \mathrm{ml} / \mathrm{min}$. A Waters $2690 \mathrm{HPLC}$ with an autosampler (Waters, Milford, Mass., USA) was used for automatic injection. The run cycle time for each sample was 2-3 min from injection to injection. A designed MS/MS program for automatic profiling of acylcarnitines and amino acids was used. The NeoLynx program for Neonatal Screening (Micromass) was used for automatic detection of abnormal samples. The cutoff levels and reference values were based on the mean concentration or peak ratio \pm standard deviation values of selected metabolites measured in 500 normal Kuwaiti babies. The values obtained were compared with those reported in other studies. The discriminative power of the NeoLynx program for automatic detection of abnormalities was tested by analyzing samples of normal and known sick patients in a blind manner. All sample results together with newborn or infant demographic data were stored in the database.

\section{Validation and Stability Studies}

Stock solutions of the diagnostic amino acids (phenylalanine, tyrosine, methionine, leucine and citrulline) and acylcarnitines (octanoylcarnitine and palmitoylcarnitine) were separately prepared in methanol/water (1:1) at concentrations of $1 \mathrm{mg} / \mathrm{ml}$. Blood samples supplemented with known concentrations of amino acids or acylcarnitines, which simulate normal and abnormal sick samples, were prepared. Blood samples containing phenylalanine at a concentration of $60-600 \mu \mathrm{mol} / \mathrm{l}$, tyrosine at $55-330 \mu \mathrm{mol} / \mathrm{l}$, methionine at 65-585 $\mu \mathrm{mol} / \mathrm{l}$, leucine at 300-900 $\mu \mathrm{mol} / \mathrm{l}$, citrulline at 55-165 $\mu \mathrm{mol} / \mathrm{l}$, octanoylcarnitine at 7-14 $\mu \mathrm{mol} / \mathrm{l}$ and palmitoylcarnitine at $10-20 \mu \mathrm{mol} / \mathrm{l}$ were used. A $50-\mu \mathrm{l}$ aliquot of each blood sample was spotted on the circle of a Guthrie card and left to dry. For validation and stability studies, five Guthrie cards of each compound were prepared, dried, stored in plastic envelops and kept at room temperature for 10 weeks. The extraction and derivatization processes were done as previously mentioned. The concentrations of the diagnostic amino acids and acylcarnitines 


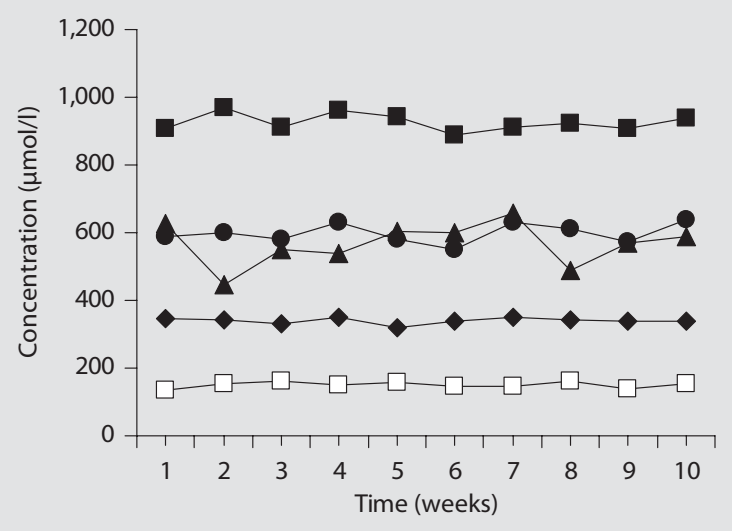

Fig. 1. Stability profiles of selected diagnostic amino acids in dried blood spots determined by MS/MS. $\mathbf{\square}=$ Leucine; $\mathbf{O}=$ phenylalanine; $\boldsymbol{\Delta}=$ methionine; $\boldsymbol{\nabla}$ = tyrosine; $\square=$ citrulline.

were measured and compared to the supplemented concentration values (table 1). The NeoLynx program for neonatal screening was used to determine the concentration of the diagnostic metabolites in blood spots $(\mu \mathrm{mol} / \mathrm{l})$ using an isotopically labeled mixture of the diagnostic acylcarnitines and amino acids as internal standard.

\section{Results}

The percent relative standard deviation and percentage deviation from the nominal concentration ranges were $2.6-14.7$ and -23.0 to $+25.0 \%$, respectively, as shown in table 1. Stability studies indicated that the analytes were stable in the dried blood spots over a 10 -week period when stored in plastic envelopes at $22 \pm 1{ }^{\circ} \mathrm{C}$ and relative humidity of $50-60 \%$ (fig. 1, 2). Of 1,158 tested newborns, 32 samples showed metabolic abnormalities, while out of 362 tested sick infants, 27 samples demonstrated positive results (table 2). Out of the total 1,520 samples screened, a total of 21 cases were previously diagnosed by another screening technique and were confirmed by MS/MS, whereas 38 positive cases ( 32 newborns and 6 sick infants) were considered undiagnosed cases and were detected by the developed MS/MS. The detected individual metabolic disorders are listed in table 2. Fatty acid oxidation defects such as very-long-chain acyl CoA dehydrogenase deficiency, long-chain hydroxyacyl CoA dehydrogenase deficiency and carnitine palmitoyl transferase type II deficiency (carnitine-acylcarnitine translocase deficiency) were the most frequently detected disorders

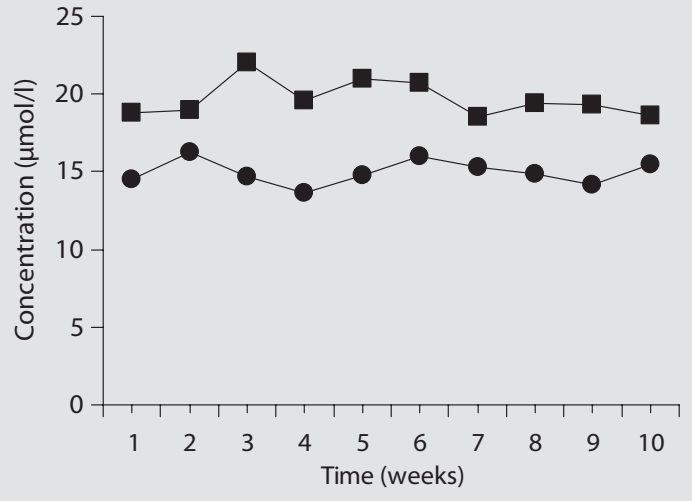

Fig. 2. Stability profiles of the diagnostic acylcarnitines in dried blood spots determined by MS/MS. $\mathbf{\square}=$ Palmitoylcarnitine; $\mathbf{O}=$ octanoylcarnitine.

Table 1. Precision and accuracy studies for the determination of some diagnostic amino acids and acylcarnitines in quality control blood spots by MS/MS

\begin{tabular}{lccrc}
\hline $\begin{array}{l}\text { Amino acid/ } \\
\text { acylcarnitine }\end{array}$ & $\begin{array}{l}\text { Nominal } \\
\text { concentra- } \\
\text { tion, } \mu \mathrm{mol} / \mathrm{l}\end{array}$ & Means $\pm \mathrm{SD}^{*}$ & \%RSD & DEV\% \\
\hline Phenylalanine & 60 & $68.0 \pm 4.4$ & 6.4 & +2 to +25 \\
& 600 & $598.0 \pm 28.9$ & 4.8 & -18 to +18 \\
\hline Tyrosine & 55 & $53.0 \pm 7.8$ & 14.7 & -23 to +20 \\
& 330 & $340 \pm 8.9$ & 2.6 & -3 to +6 \\
\hline Methionine & 65 & $69 \pm 8.7$ & 12.6 & -15 to +11 \\
& 585 & $568 \pm 63.1$ & 11.1 & -23 to +12 \\
\hline Leucine & 150 & $141 \pm 10.1$ & 7.2 & -12 to +7.5 \\
\hline Citrulline & 900 & $926 \pm 25.9$ & 2.8 & -2.0 to +8 \\
\hline Octanoyl- & 55 & $57 \pm 4.8$ & 8.7 & -11 to +15 \\
carnitine & 14 & $150 \pm 9.1$ & 6.1 & -1.5 to -19 \\
\hline Palmitoyl- & 10 & $12.4 \pm 1.1$ & 8.9 & -11 to +20 \\
carnitine & 20 & $19.6 \pm 1.2$ & 5.9 & -7.5 to +10 \\
\hline
\end{tabular}

$\% \mathrm{RSD}=$ Percent relative standard deviation; $\mathrm{DEV} \%=$ percent age deviation from nominal concentration. ${ }^{*} \mathrm{n}=10$.

among newborns and symptomatic infants. The MS/MS profile of a newborn with a significantly high concentration of palmitoylcarnitine (C16) at $\mathrm{m} / \mathrm{z} 456$ is shown in figure 3. Organic acid disorders such as propionic and 
Table 2. Screening for IMDs by tandem mass spectrometry in 1,520 samples collected from newborns and symptomatic infants

\begin{tabular}{|c|c|c|c|c|}
\hline \multirow[t]{2}{*}{ Metabolic disorder } & \multicolumn{2}{|c|}{$\begin{array}{l}\text { Diagnosis prior } \\
\text { to MS/MS }\end{array}$} & \multicolumn{2}{|c|}{ Screening $(1,520)$} \\
\hline & yes & no & $\begin{array}{l}\text { symptomatic } \\
\text { infants (362) }\end{array}$ & $\begin{array}{l}\text { newborns } \\
(1,158)\end{array}$ \\
\hline \multicolumn{5}{|l|}{ Amino acid disorders } \\
\hline Transient tyrosinemia & & $\mathrm{x}$ & & 7 \\
\hline Tyrosinemia type I & & $\mathrm{x}$ & & $1(1 \mathrm{ND})$ \\
\hline Phenylketonuria & $\mathrm{x}$ & & 1 & \\
\hline Hyperphenylalaninemia & & $\mathrm{x}$ & & 3 \\
\hline Maple syrup urine disease & & $\mathrm{x}$ & & 1 \\
\hline Homocystinuria & $\mathrm{x}$ & & 1 & \\
\hline Non-ketotic hyperglycinemia & & $\mathrm{x}$ & & 1 \\
\hline \multicolumn{5}{|l|}{ Hyperalaninemia secondary to } \\
\hline pyruvate carboxylase deficiency & & $\mathrm{x}$ & & $3(1 \mathrm{ND})$ \\
\hline \multicolumn{5}{|l|}{ Organic acid disorders } \\
\hline Propionic acidemia & $\mathrm{x}$ & $\mathrm{x}$ & $3^{1}$ & \\
\hline Methylmalonic acidemia & $\mathrm{x}$ & $\mathrm{x}$ & $2^{1}$ & \\
\hline Isovaleric acidemia & $\mathrm{x}$ & & 1 & \\
\hline Glutaric acidemia type I & $\mathrm{x}$ & $\mathrm{x}$ & $1^{1}$ & \\
\hline Hydroxymethyl glutaric acidemia & $\mathrm{x}$ & & 1 & \\
\hline \multicolumn{5}{|l|}{ Fatty acid oxidation defects } \\
\hline \multicolumn{5}{|l|}{ Very-long-chain acyl CoA } \\
\hline dehydrogenase deficiency & & $\mathrm{x}$ & 3 & $5(1 \mathrm{ND})$ \\
\hline \multicolumn{5}{|l|}{ Long-chain hydroxyacyl CoA } \\
\hline dehydrogenase deficiency & & $\mathrm{x}$ & & $3(1 \mathrm{ND})$ \\
\hline \multicolumn{5}{|l|}{ Medium-chain acyl CoA } \\
\hline dehydrogenase deficiency & & $\mathrm{x}$ & 2 & 2 \\
\hline Multiple acyl CoA dehydrogenase deficiency & & $\mathrm{x}$ & & $1(1 \mathrm{ND})$ \\
\hline \multicolumn{5}{|l|}{ Carnitine palmitoyl transferase } \\
\hline Type I deficiency & & $\mathrm{x}$ & 1 & \\
\hline Type II deficiency & & $\mathrm{x}$ & & $3(2 \mathrm{ND})$ \\
\hline \multicolumn{5}{|l|}{ Urea cycle disorders } \\
\hline Citrullinemia & & $\mathrm{x}$ & 2 & 2 \\
\hline Argininosuccinic acidurias & $\mathrm{x}$ & & 2 & \\
\hline \multicolumn{5}{|l|}{ Others } \\
\hline Canavan's disorder & $\mathrm{x}$ & & 1 & \\
\hline Cases & & & 27 & 32 \\
\hline Previously diagnosed & & & 21 & 0 \\
\hline Diagnosed by MS/MS only & & & 6 & 32 \\
\hline Newborn deaths (ND) & & & & 7 \\
\hline
\end{tabular}

${ }^{1}$ Infants were previously undiagnosed and only detected by MS/MS. methylmalonic acidemia were common among symptomatic infants. A similar MS/MS profile of a sick infant with a distinct high level of propionylcarnitine (C3) at $\mathrm{m} / \mathrm{z} 275$ is shown in figure 4 . A very rare case of Canavan's disorder was detected in the urine sample of 1 sick infant (fig. 5). The profile shows a significantly increased concentration of $\mathrm{N}$-acetylaspartate at $\mathrm{m} / \mathrm{z} 288$. Generally, the obtained concentrations of the metabolites in sick infants were much higher than the normal values. Seven newborn deaths were reported due to fatty acid oxidation defects, pyruvate carboxylase deficiency and tyrosinemia type I. 
Fig. 3. Blood spot of a newborn with carnitine palmitoyltransferase type-II deficiency determined by MS/MS. C16: $11.3 \mu \mathrm{mol} / \mathrm{l}$ (normal range $1.5-2.5 \mu \mathrm{mol} / \mathrm{l}$ ).
180605-009 1 (2.032)

$\%$

100

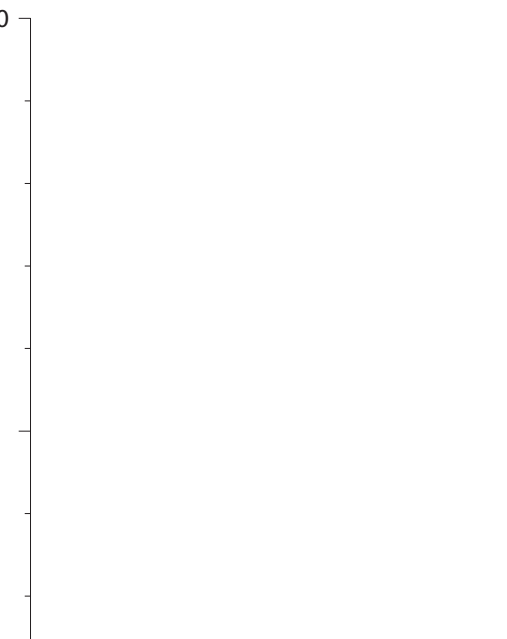

1: Parents of $85 E S+1.61 e 6$

Palmitoylcarnitine (C16)

456.20

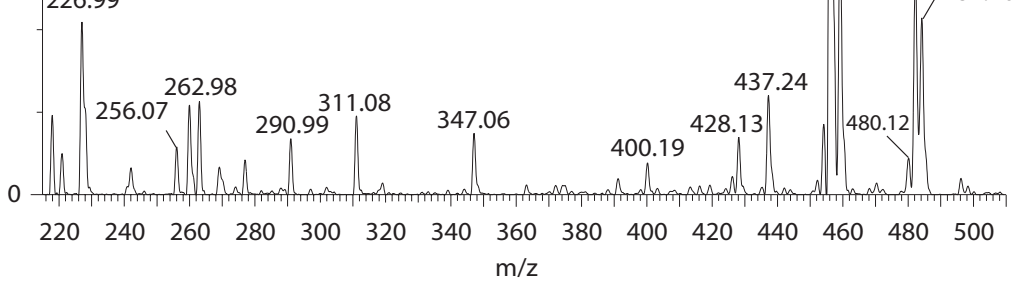

030504-001 1 (2.032)

1: Parents of $85 \mathrm{ES}+7.44 \mathrm{e} 5$

$\%$

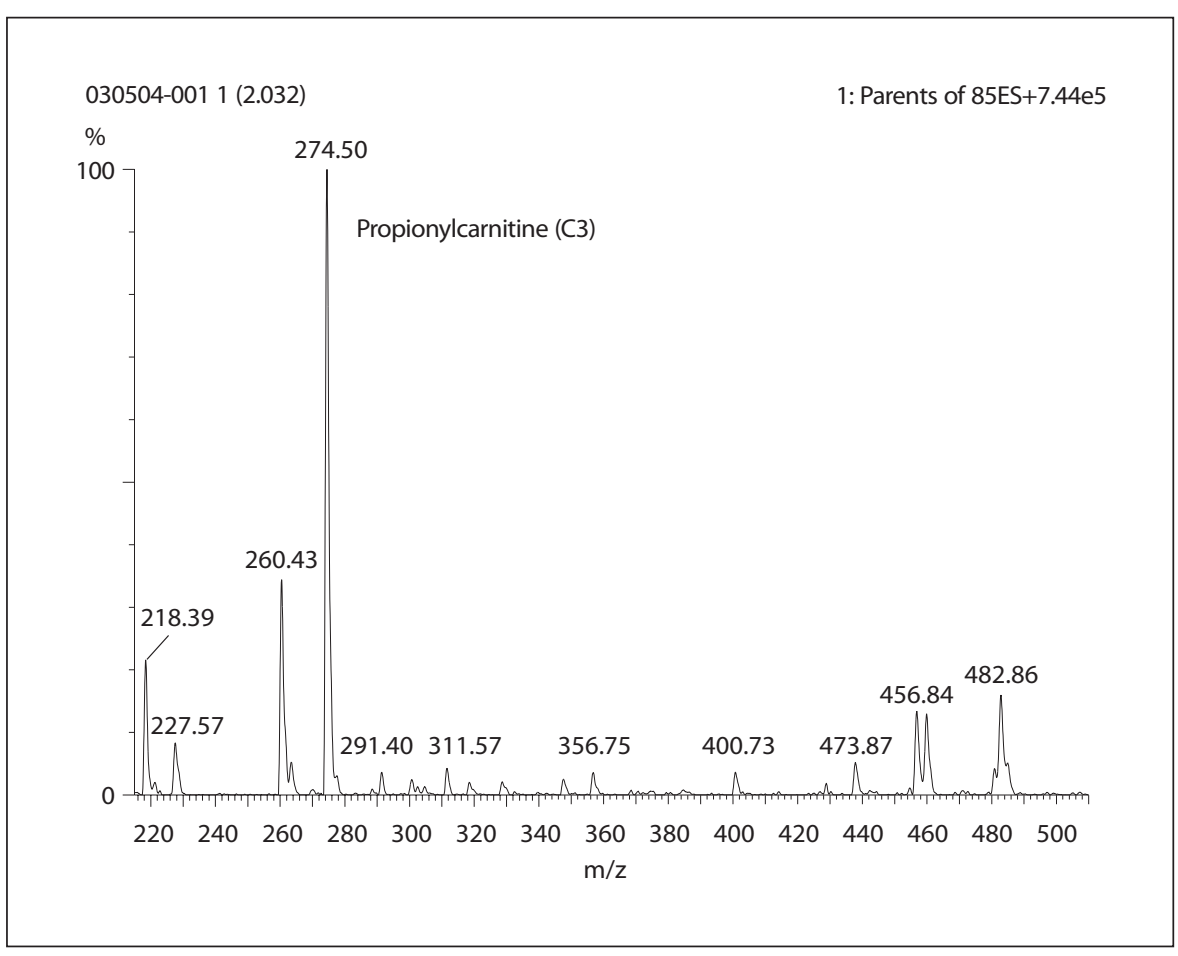

Fig. 4. Blood spot of a sick infant with propionic acidemia indicated by MS/MS. C3: $35.5 \mu \mathrm{mol} / \mathrm{l}$ (normal range 1.0 $1.5 \mu \mathrm{mol} / \mathrm{l})$. 
Fig. 5. Urine sample of a sick girl with Canavan's disorder detected by MS/MS. $\mathrm{N}$-acetylaspartate (NAASP; a marker of Canavan's disease): $235 \mu \mathrm{mol} / \mathrm{l}$ (normal range $20-25 \mu \mathrm{mol} / \mathrm{l}$ ).

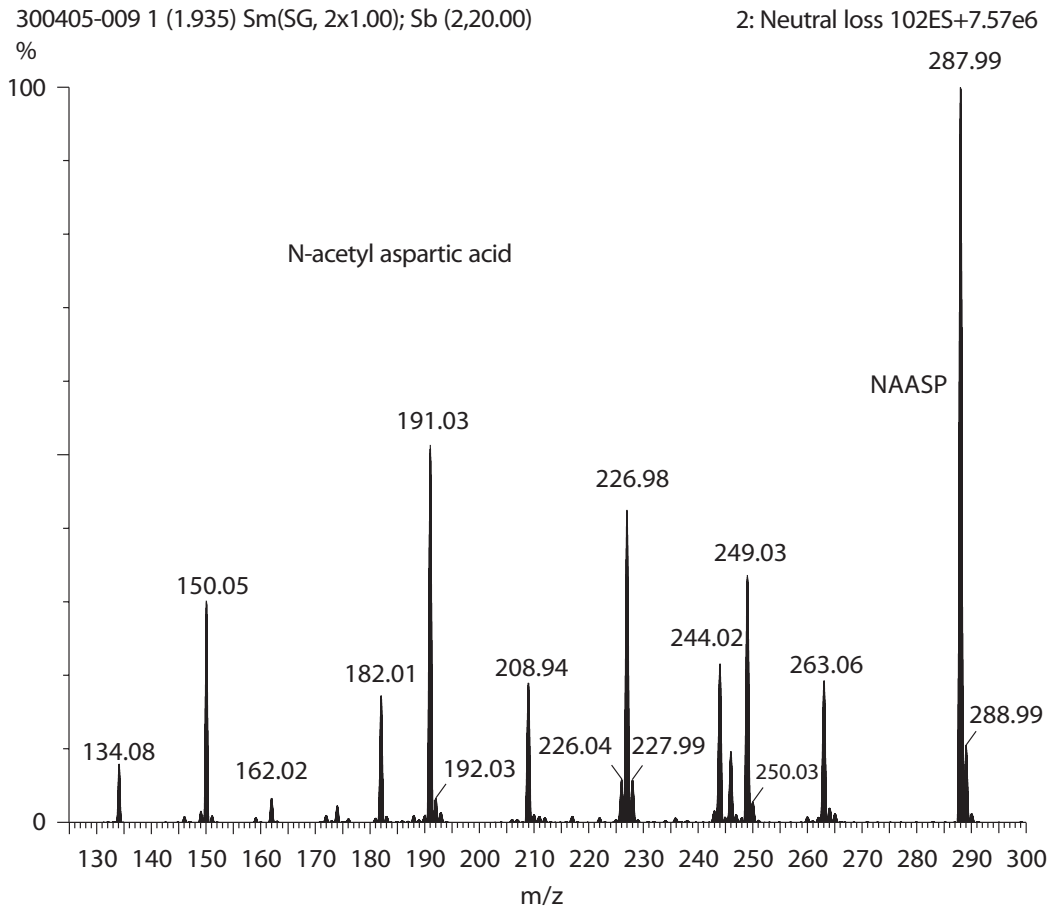

$\mathrm{m} / \mathrm{z}$

\section{Discussion}

In pediatric practice, early diagnosis of IMDs in newborns and young infants is significantly important to avoid serious consequences such as mental retardation and developmental delay. In clinical metabolic screening laboratories, many screening techniques are applied such as ion-exchange chromatography [13], thin-layer chromatography, paper electrophoresis [14] and gas chromatographic methods $[15,16]$. The drawbacks of these techniques are: low sensitivity and specificity, narrow spectrum of diseases detected, extensive sample preparation, too long chromatographic run time and a high rate of false-positive results. Furthermore, these techniques are not capable of detecting serious fatty acid oxidation disorders. Metabolic screening using tandem mass spectrometry permits a fast, sensitive and specific screening for IMDs. Equally important, the technique has high sample throughput which permits analysis of a large number of samples ( $>100$ samples) in few hours. Consequently MS/MS enables the detection of diagnostic levels of amino acids and acylcarnitines in blood spots; in addition, more than 20 IMDs can be detected with a high degree of specificity and sensitivity in a single run. Optimization of the experimental and instrumental conditions is necessary to achieve optimum measurement of metabolites in blood samples. Prior to the application of the developed MS/MS to patient samples, the validity of the method for analysis of the target metabolites was evaluated. Quality control blood spots containing the diagnostic metabolites at normal and abnormal concentrations were analyzed by the developed MS/MS procedure using an isotopically labeled internal standard mixture. The calculated percent relative standard deviation and percentage deviation from nominal concentration values were appropriate and proved the reproducibility and accuracy of the method. Further, the stability of the diagnostic metabolites in blood spots, during sample collection and storage, was assessed by replicate analysis of quality control blood samples over a 10 -week period. The calculated concentrations fluctuated within $10-15 \%$ concentration ranges. Application of the developed MS/MS to the analysis of blood samples collected from Kuwaiti newborns and symptomatic 
infants from different governmental maternity and pediatric hospitals in Kuwait indicated high rates of IMDs (table 2). Fatty acid oxidation defects such as very-longchain acyl CoA dehydrogenase deficiency and carnitine-palmitoylcarnitine transferase deficiency and organic acid disorders such as propionic and methylmalonic acidemias were clearly recognized. The robustness of MS/MS technology enables the detection of these metabolic abnormalities with high certainty. The obtained results suggest the justification of the establishment of a national newborn screening program in $\mathrm{Ku}-$ wait using tandem mass spectrometry to avoid the burden of neurometabolic diseases and the associated long-term care on the health system. Although newborn screening by tandem mass spectrometry is more expensive than other screening techniques, the screening program is beneficial and is likely cost saving to society in the long term [17].

\section{Conclusion}

Tandem mass spectrometry is a powerful technology that facilitates an early diagnosis of more than twenty IMDs in newborns and sick infants. The utility of the MS/MS technique in a newborn screening program and as a high-quality clinical service for metabolic screening in maternity and pediatric hospitals in Kuwait is highly recommended.

\section{Acknowledgments}

The authors are grateful to the Research Administration, $\mathrm{Ku}$ wait University, for the financial support through Research Grant $\mathrm{PC} 02 / 02$. They are also thankful to the Faculty of Pharmacy, Kuwait University, for providing the facility of MS/MS used throughout this work. The authors highly appreciate the cooperation of metabolic specialists and pediatricians in the Kuwait Medical Genetic Center, and maternity and pediatric hospitals in Kuwait. They are also indebted to the Secretary General of the Kuwait University for his kind approval to provide the necessary funds to continue the clinical service of metabolic screening at the Faculty of Pharmacy, Kuwait University.

\section{References}

1 Seashore MR, Seashore CJ: Newborn screening and the pediatric practitioner. Semin Perinatol 2005;29:182-188.

-2 Rinaldo P, Tortorelli S, Marten D: Recent developments and new applications of tandem mass spectrometry in newborn screening. Curr Opin Pediatr 2004; 16:427-433.

$\checkmark 3$ Cheillan D, Cognat S, Vianey-Saban C, Maire I, Dorche C: Application of tandem mass spectrometry to neonatal screening of inherited metabolic diseases: focus on present developments (in French). Ann Biol Clin (Paris) 2004;62:269-277.

4 Wilcken B, Wiley V, Hammond J, Carpenter $\mathrm{K}$ : Screening newborns for inborn errors of metabolism by tandem mass spectrometry. N Engl J Med 2003;348:2304-2312.

5 Schulze A, Lindner M, Kohlmuller D, Olgemoller K, Mayatepek E, Hoffman GF: Expanded newborn screening for inborn errors of metabolism by electrospray ionizationtandem mass spectrometry: results, outcome and implications. Pediatrics 2003;111:13991406.

6 Shigematsu Y, Hirano S, Hata I, Tanaka Y, Sudo M, Sakura N, Tajima T, Yamaguchi S: Newborn mass screening and selective screening using electrospray tandem mass spectrometry in Japan. J Chromatogr B Analyt Technol Biomed Life Sci 2002;776:39-48.
7 Rashed MS: Clinical applications of tandem mass spectrometry: ten years of diagnosis and screening for inherited metabolic diseases. J Chromatogr B Biomed Sci Appl 2001; 758:27-48.

8 Rashed MS, Bucknall MP, Little D, Awad A, Jacob M, Alamoudi M, Alwattar M, Ozand PT: Screening blood spots for inborn errors of metabolism by electrospray tandem mass spectrometry with a microplate batch process and a computer algorithm for automated flagging of abnormal profiles. Clin Chem 1997;43:1129-1141.

-9 Zytkovicz TH, Fitzgerald EF, Marsden D: Tandem mass spectrometric analysis for amino, organic and fatty acid disorders in newborn dried blood spots: a two-year summary from the New England Newborn Screening Program. Clin Chem 2001;47: 1945-1955.

10 Al-Aqeel A: Common genetics and metabolic diseases in Saudi Arabia Middle East. J Fam Med 2004, p 6.
11 Joshi SN, Hashim J, Venugopalan P: Pattern of inborn errors of metabolism in an Omani population of the Arabian Peninsula. Ann Trop Paediatr 2002;22:93-96.

$\checkmark 12$ Meyer BF: Strategies for the prevention of hereditary diseases in a highly consanguineous population. Ann Hum Biol 2005;32:174179.

13 Suh JW, Lee SH, Chung BC: GC-MS determination of organic acids with solvent extraction after cation-exchange chromatography. Clin Chem 1997;43:2256-2261.

14 Slocum RH, Cummings JG: Amino acid analysis of physiological samples; in Hommes FA (ed): Techniques in Diagnostic Human Biochemical Genetics. New York, Wiley-Liss, 1999, pp 87-126.

15 Shih VE: Amino acids. Laboratory Techniques for the Detection of Hereditary Metabolic Disorders. Cleveland, CRC Press, 1973, pp 11-76.

16 Tanaka K, West-Dull A, Hine DG, Lynn TB, Lowe T: Gas-chromatographic method of analysis for urinary organic acids. II. Description of the procedure, and its application to diagnosis of patients with organic acidurias. Clin Chem 1980;26:1847-1853.

17 Carroll AE, Downs SM: Comprehensive cost-utility analysis of newborn screening strategies. Pediatrics 2006;117:S278-S295. 